\title{
EDUCACIÓN PARA LA PAZ Y PARA LA DEMOCRACIA
}

\author{
Pedro Ceballos Rendón
}

\section{Resumen}

La construcción de la paz y la democracia marchan paralelamente, ambas tienen que ser construidas mediante procesos de educación que han de: (1) respetar la historicidad en que se definió la categoría de ciudadanía (2) propiciar que la sociedad tenga acceso a información veraz e imparcial, (3) permitir espacios de expresión abierta al diálogo y (4) rescatar las figuras de autoridad como formadores de las nuevas generaciones dentro de un marco ético que instaure al otro como un semejante, sujeto también a derechos y obligaciones que ambos habrán de respetar, dando paso a la tolerancia y a la convivencia pacífica.

Palabras clave: sujeto social, ciudadanía, democracia, diálogo, paz

\section{Abstract}

The construction of peace and democracy march in parallel, both have to be constructed by processes of education they have to: (1) respect the historicity that defined the category of citizenship, (2) encourage society to have access to real and impartial information, (3) allow expression spaces open to dialogue y (4) rescue authority figures as forming new generations within an ethical framework to put in place the individual as a fellow citizen, with rights and obligations that both have to be respected, leading to tolerance and peaceful 
coexistence.

\section{EDUCACIÓN PARA LA PAZ - EDUCACIÓN PARA LA DEMOCRACIA}

"En la actualidad la Educación para la Paz y los Derechos Humanos -concebida en su triple finalidad de informar, formary transformar, constituye un importante instrumento de construcción de la nueva cultura que la humanidad anhela. (...) La educación para la paz asimilada e integrada transversalmente por algunas reformas educativas, no debe desviarse de su verdadera razón de ser: desaprender constantemente las consignas de una cultura basada en la intolerancia, la competitividad, la insolidaridad y el belicismo."

José Tuvilla Rayo

La conjunción de la educación para la pazy la educación para la democracia obedece a la convicción de que tanto la paz como la democracia, son construcciones sociales que marchan juntas en la edificación de una sociedad lo suficientemente madura para lograr un adecuado grado de desarrollo humano de los ciudadanos y de garantía de los derechos humanos; pero, como construcciones, se trata de procesos que pueden y deben ser amparados por el trabajo formativo de la educación como una política social.

Llegar a un estado pacífico, de paz activa (esto es, la paz que se construye transformando las condiciones sociopolíticas), solo es posible bajo el cobijo de la democracia, como lo explica Solís (1955) en una cita extraída del texto Educación para la paz.

"... es conveniente adoptar una visión integral de la paz como fenómeno social y político. Es decir, que la experiencia humana ha demostrado que múltiples son los caminos hacia el establecimiento de una sociedad pacífica y equitativa; y que lo importante más que el predominio de una teoría sobre otra, es la satisfacción de ciertos principios básicos entre los que destacan el respeto a los Derechos Humanos y la preservación de la vida como sustento mismo de la convivencia civilizada.

En este sentido, no es posible concluir este acápite sin adoptar una posición ética. A nuestro juicio, la consecución de la paz -en su acepción más integralno es posible sin el perfeccionamiento de los procesos democráticos. Estos procesos, que están condicionados por la experiencia histórica de cada pueblo o nación, conllevan, como signo distintivo, la promoción de la libertad y la búsqueda de la equidad económica y social para el mayor número. En otras palabras, la paz sólo se puede garantizar en el largo plazo en sociedades justas, pluralistas, participativas y prósperas". (Solís, et al, 1995: 52-3).

Educar para la paz requerirá de una educación para la democracia: son una diada inseparable desde un mismo núcleo: la tolerancia y el respeto al otro y a su lugar en el espectro social.

\section{EDUCACIÓN, PAZ Y DEMOCRACIA}

Aguilera define la Pazcomo

“...el proceso de realización de la justicia en los distintos niveles de relación humana. Es un concepto dinámico que nos lleva a hacer aflorar, afrontar y resolver conflictos de forma noviolenta y cuyo fin es el logro de una armonía de la persona consigo misma, con la naturaleza y con los demás" (Aguilera et al, 2000: 16)

Aceptando este constructo, entendemos que prácticamente todos los espacios de la relación humana pueden presentar riesgos de llevar los vínculos entre los sujetos de formas poco pacíficas e incluso violentas, pero también da paso a la idea de favorecer la formación de sujetos a favor de la paz es requerido en términos de competencia social, construyendo recursos por los que el sujeto pueda usar su inteligencia y su emoción en pro de vivir vínculos buscando acuerdos con los semejantes por quienes se acompaña. Al respecto, y citado por Monclús y Saban, Federico Mayor Zaragoza, Presidente de la Fundación Cultura de Paz sostiene que:

"La educación debe proporcionar herramientas para que los ciudadanos entiendan el complejo mundo en el que viven, lo gestionen democráticamente, usen equilibradamente los recursos naturales y construyan y defiendan un sistema de valores en el que estén integrados la tolerancia, la justicia, el respeto a las diferencias" (Monclús et al, 2008: 12)

Antonio Monclús (2008) titula la primera parte de su texto La paz como centro de la Educación y la Ciudadanía, es indudable que estos tres conceptos Paz-Educación-Ciudadanía están fuertemente entrelazados, generando incluso una relación de interdependencia, a lo que es posible agregar que la formación ciudadana en pro del desarrollo de una cultura de la paz, deberá tener como punto angular la aceptación de los derechos y prerrogativas del otro, de modo que está íntimamente ligado a la condición de construir 
ciudadanos democráticos

Monclús y Saban (2008:40) citan a Carlos Rosales López quien en su texto Cuestiones de interés universal señala que "La educación constituye un estímulo a la democracia y la cultura de la paz al hacer a las personas críticas y libres, capaces de adoptar decisiones". Pero, ¿cuál es el punto de engarce entre educación para la paz y educación para la democracia? Para analizar este asunto, partiremos del tema de la participación política, cuestión de abordaje obligatorio, especialmente en un país que recién está dando visos de ir desarrollando una cultura democrática y a la que aqueja una alta incidencia de actos de violencia; de modo que, ya en el inicio de un tercer milenio ahora globalizado en el que estamos como país cada vez más frecuentemente en una estrecha relación con pueblos y culturas preocupados y ocupados actuando como sujetos de la política y sujetos en búsqueda de la paz tanto al seno de su mismo pueblo como hacia fuera del mismo, es casi natural la inquietud de muchas instancias y personalidades, las preguntas de ¿Por qué la gente no participa en los asuntos de la política?, ¿Por qué una gran mayoría no están atendiendo, desde su condición de ciudadanos, la construcción de una sociedad pacífica?, ¿Cuáles son las motivaciones que tienen la fuerza de llevar al ciudadano a participar en la definición de la organización de la vida pública? ¿Cómo favorecer que la participación cívica y política se transforme de una posibilidad a un imperativo personal en pro del afán de ganar democracia y paz social?

\section{LA HISTORICIDAD DEL CIUDADANO}

Para empezar este análisis es menester hacer un reconocimiento de lo histórico en el orden de lo sociocultural: los países cuyos ciudadanos tienen una conciencia más o menos generalizada de la trascendencia del propio actuar político, son los que como pueblo han venido por un complejo y las más de las veces largo período de adquisición de la conciencia social y de la posibilidad de intervenir en asuntos comunitarios, proceso que en su transcurrir, han llevado a generar un clima propicio a la lucha por que la voz del pueblo se oiga, ganando el derecho de tomar parte en las decisiones del quehacer en la organización social. Es sabido que un amplio margen de estas ganancias lo han sido por vías violentas, de modo que la educación para la paz y la democracia tiene aquí un gran espacio de oportunidad: la construcción de recursos en los ciudadanos para buscar el desarrollo social por vías pacíficas.

En el histórico desarrollo de un pueblo, se va dando forma paulatinamente a la cultura del mismo, estructurándose un conjunto de conocimientos, actividades e ideas que le caracterizan como ciudadanía, más allá del carácter jurídico y por supuesto que su organización cultural tiene peso en las actitudes de los sujetos que de ella participan. En nuestro país, a pesar de un transcurrir histórico de guerras, guerrillas, revoluciones y revueltas en la lucha por la estructuración de un gobierno que resuelva de mejor manera los problemas de las comunidades en particular y de la nación en general, la conformación de una cultura primordialmente democrática está aún en ciernes aunque ya va dando visos del desarrollo y funcionamiento de movimientos sociales que buscan de hacer de la manifestación pacífica su voz para dar cuenta de la inconformidad y el deseo de vivir en una sociedad diferente, como los plantones del Zócalo de la Ciudad de México ante la duda en la validez del resultado electoral en el 2006 y las marchas en protesta por la violencia y a favor de la paz en diversas ciudades de nuestro país durante los últimos dos años y más recientemente las manifestaciones del grupo de jóvenes "Yo soy 132". Incluso es posible ya dar cuenta organizaciones de ciudadanas que tratan de hacer del diálogo la forma privilegiada de lograr nuevos acuerdos, como el Movimiento por la paz con justicia y dignidad, encabezado por el poeta Javier Sicilia.

Sin embargo, aún distamos como país de ostentarnos con las categorías de "pacífico" y "demócrata", en este último tenor de ideas, apenas estamos saliendo de un régimen presidencialista que favoreció el desarrollo de una cultura política en la que se tenía conciencia del autoritarismo ${ }^{1}$ gubernamental que se resistía a permitir la alternancia. Este presidencialismo regía desde los espacios sociales hasta los familiares; recordemos que no hace muchas décadas el patriarca familiar acudía a la casilla de votación con las credenciales electorales de los miembros de su familia a emitir su voto en elecciones constitucionales a nombre de los títulos portados. Igualmente en los espacios gubernamentales este sistema presidencialista tiene fuertes efectos en la cultura política: En más de la última mitad del siglo pasado, el régimen político de México funcionó con un presidente de amplio poder político, y grupos institucionales organizados en torno a esta figura presidencial, sistema que se prolongaba a sí mismo mediante estructuras constitucionales que otorgaron 
una legal continuación a este tipo de régimen gubernamental.

Si intentamos llegar a un entendimiento en cuanto a en qué términos se complicó más este escenario para la construcción de un ambiente de paz y democracia, me queda a la mano pensar que es la intolerancia. Es en ella donde no hay lugar al respeto hacia el otro o se hace imposible la comprensión de las acciones ajenas. El sujeto es incapaz de asimilar y dar cabida a lo que le es ajeno y al ser enfrentado a la evidencia de la existencia de ese otro que pone en riesgo la posesión de sus objetos de goce, se producen reacciones correspondientes a una sensibilidad extrema y contrariedad, que dan vida a actitudes defensivas y a posturas que se exhiben apasionadamente, llegando a obnubilar la racionalidad, perturbando un encuentro amable, por ejemplo, en las pugnas ideológico políticas, cada uno se esfuerza en hacer que se acepte la oferta propia y anular la propuesta ajena.

El compromiso a la tolerancia mostrada en el respeto por la pluralidad ideológica política es un aspecto que idealmente habría de ser acatado en los espacios democráticos; aún en los tiempos actuales si bien en el discurso político pareciera siempre haber un lugar distinguido para convivir pacíficamente con quienes tienen una postura diferente a la propia, en lo concreto la intolerancia es un acontecer de lo cotidiano y la pregunta hacia las ciencias sociales sería: el porqué de la intolerancia de un amplio número de los actores políticos, tanto de la sociedad en general como de los que directamente tienen o aspiran al poder político.

\section{EDUCANDO SE CONSTRUYE LA CATEGORÍA CIUDADANA PARA} LA CONSTRUCCIÓN DE LA PAZ SOCIAL

Si bien la actual cultura política da lugar a distintas formas de participación ciudadana, ésta tiene que ir acompañada de un proceso de educación por el cual vaya quedando clara la trascendencia del ejercicio ciudadano de participar, entendido como un derecho para involucrarse en las decisiones comunitarias de manera tan activa como pacífica, respetuosa siempre del otro o los otros.

Se entiende la ciudadanización como un proceso por medio del cual el sujeto que pertenece a una comunidad organizada, toma conciencia de que tiene determinados derechos y deberes públicos, que son compartidos con otros iguales que él, así se instaura en su condición de con-ciudadano, actuando en consecuencia de manera individual y/o colectiva, pero siempre sabiéndose y viviéndose como parte activa de un colectivo, el que él mismo es tanto causa como efecto.

En estos términos, el proceso de ciudadanización es como el sujeto va siendo tomado por lo comunitario, como va apropiándose de un lugar en el espectro de lo social y en relación con el resto de los conciudadanos erigiéndose como un sujeto de la política, quien tiene opinión acerca de la organización de la administración pública y quien puede mostrar su criterio en el discurso y en los actos, esto es, su intervención en asuntos ciudadanos que reclaman su atención, como en su participación política electoral, en la definición de las instituciones de lo político, en el sistema partidista, en los fenómenos de las masas en los actos de proselitismo electoral, en su concepción de la democracia, en el derecho electoral, en la historia de las elecciones, en el abstencionismo, en el financiamiento de las campañas y precampañas, en la tolerancia, en la diversidad ideológico-política, etc.

\section{EL SUJETO DE LO SOCIAL}

Tenemos entonces esta dimensión del análisis en términos de conciencia social, de lo que socioculturalmente implica el existir como un ser social, tomando conocimiento y actuando en consecuencia de ello, interviniendo en asuntos públicos, de modo que desarrollar este saberse sujeto de lo social se convierte en un motivo para encontrar sentido práctico a la participación política, ya que con este proceso de socialización es donde se vienen generando actitudes a través de información y demás símbolos que se intercambian con los grupos en los que se participa, empezando en la familia, pasando por la escuela y el total de prácticas sociales que el sujeto vive.

Este proceso socializador-ciudadanizador del sujeto debe darse cobijado por los principios éticos y morales del "ser político-social", que impulsen una participación respetuosa, de buenos juicios, mediada por la reflexión, la racionalidad y la conciencia de las situaciones objetivas por las cuales regir la conducta propia. Luego de tomar conciencia y postura como "ser social", es factible que aparezca el desarrollo de la identidad cultural como una condición que favorece en alta medida el interés por participar en asuntos concernientes a definir la organización social en términos pacíficos y democráticos. 


\section{LA INFORMACIÓN COMO EDUCACIÓN CONTÍNUA DE LA} CIUDADANÍA

Existen áreas donde la racionalidad puede tener un mayor juego y es en los espacios de discusión y análisis políticos (incluida aquí los debates en torno a los procesos sociales de violencia y de construcción de la paz), aquí está la trascendencia de que el acceso a la información sea un derecho irrestricto, al menos en cuanto se refiera a la estructura e ideología de las formas de organizar la práctica de la política.

Se señala aquí la trascendencia de la información objetiva y amplia para la elaboración de juicios que respondan cada vez en mayor medida a las condiciones de la realidad. Las instituciones deben de apostar más a la inteligencia ciudadana, confiar en el funcionamiento de sus procesos para pensar, elaborar juicios sociales y actuar en consecuencia.

Se localiza en el pensamiento del ciudadano la dimensión racional y emotiva del mismo en donde lo racional será el orden de las ideas que eso solo es como dar luz a la meta de lo que se busca, para eso sirven las ideas y la emoción es la energía que hace que esas ideas tengan vida y en la práctica se luche por ellas y en esa conjunción es donde podemos encontrar propiamente la motivación para ir por lo que la ideas reclaman y así mantener viva la participación de la ciudadanía de forma permanente alrededor de su ejercicio ciudadano, como única garantía de que las instituciones y leyes al respecto sostengan una dinámica social favorecedora del desarrollo humano y la paz social.

Una de las tareas para darle mayor impulso a la participación ciudadana es generar confianza hacia las instituciones públicas en general y especialmente en las de orden político, confianza de ser escuchados y tomados en cuenta, dando así al ciudadano la seguridad en la legalidad, la transparencia y en consecuencia el respeto y la tolerancia a sus puntos de vista.

Habremos de aceptar que este confiar se gana solamente a través de una legislación sólida y un amplio respeto por el cumplimiento de la ley en general y electoral en lo particular, solo así es que llegaremos a un modelo de organización social democrático más maduro, ahí es donde quien detenta el poder público, debe promover que las demandas de la sociedad vayan cada vez más orientadas hacia el interés general, ya que es a través de ello que se pueden promover los valores y normas que dan fortaleza al funcionamiento de la democracia y es por esa vía donde podremos consolidar hombres libres, responsables, tolerantes, informados en igualdad ante la ley, donde siempre se asuman las consecuencias de los actos que se practican y terminen siempre por sujetarse solo a la normatividad jurídica como precepto rector.

Más allá de los preceptos que el Estado debería contemplar para favorecer la construcción de identidades ciudadanas (sujetos que se identifiquen como ciudadanos) y del ejercicio cívico coadyuvado por el libre acceso a la información y la libertad de expresión como andamiajes para la construcción de la paz social, es requerida por parte del ciudadano, la paz humana y el reconocimiento del otro.

\section{PAZ SOCIAL, PAZ HUMANA Y EL RECONOCIMIENTO DEL OTRO}

Para conseguir paz social es necesario partir de un concepto de paz humana, una condición desde lo subjetivo (relativo al sujeto mismo) favorecedora del establecimiento y vivencia de vínculos interpersonales armoniosos con el semejante: Tener paz interior es requisito para la construcción de relaciones pacíficas con los semejantes.

No podemos conseguir esa paz interior y seguridad de forma autónoma ni en soledad, necesariamente necesitamos contar con el otro.

Es precisamente "ese otro" el que tiene la posibilidad, con su presencia significativa, de dar vida al sentimiento del sujeto al poner enfrente un objeto al que dirigir el afecto, que se inicia con el requisito de mirarlo, de dar cuenta que existe ahí alguien digno de ser visto, escuchado y reconocido como un semejante por quien sentirse acompañado, amenazado, valorado, minimizado, etc.

La presencia del otro en condición de un ser semejante a uno mismo, saca al sujeto de su propio ensimismamiento, puede romper el esquema narcisista, forzándolo a transitar de lo concéntrico (dirigido hacia sí mismo) a lo excéntrico (dirigido hacia afuera del sí mismo, al ambiente, a los demás).

No podemos cuidar lo propio olvidando y/o negando lo ajeno, ya que la mirada interior es vana sin el otro que es el referente del concepto que de sí mismo tiene el sujeto, por ello, es en los términos de su propia aceptación personal como puede recibir al otro con el que se encuentra.

La convivencia tiene que iniciar con optimismo y mantener la lucha para que no se desvanezca, ya que la comprensión de los problemas ayuda a entender el mundo de la interacción.

Para lograr el cambio de esas viejas actitudes, y llevar a la construcción de un 
sujeto que se sienta disminuido por el dolor ajeno así como congratulado con la buenaventura del otro, tenemos que estar convencidos de que es necesario encontrar modelos de grupos que nos den la oportunidad de establecer relaciones con los demás en las que todos podamos ganar algún trozo de identificación y que estemos conscientes que en ese aprendizaje siempre lo trascendente será interactuar con los demás, esos que compartan y respeten la necesidad del saber y en ese proceso los libros siempre serán un punto de apoyo para mover las ideas.

Con todo esto, sí será posible construir el círculo de la educación para la convivencia y convivir para educarse. Ése será el abordaje para la definición de los contextos que funcionen como nuevos nidos que favorezcan el desarrollo de lo humano, en lo que las figuras de autoridad tienen la función formativa básica.

LA FUNCIÓN DE LAS FIGURAS DE AUTORIDAD AL ERIGIR LA FIGURA DEL OTRO: CONDICIÓN PARA LA PAZ Y LA DEMOCRACIA

Las figuras de autoridad (padres, maestros y representantes de las instituciones del Estado) están en función de ser los reguladores (inhibidores, potenciadores, canalizadores, moderadores y anuladores) de las violencias, asimismo de la construcción de las condiciones psicológicas en las que se basan los vínculos armoniosos, solidarios y empáticos con el semejante, esto es, el vivirse reglamentado por ordenamientos formales e informales, incluyendo la cultura de la legalidad.

El orden que provee la legalidad define las condiciones y formas de interrelación social, ya que la ley es simbólicamente estructurante al operar definiendo prohibiciones y permisiones, regulando la convivencia en búsqueda de preservar el bien común. La instauración de este ordenamiento social en la niñez es de primordial importancia en la formación de un sujeto adaptado, respetuoso y productivo, así como le otorga la posibilidad de vivir en paz con quienes le rodean.

La violencia que hoy por hoy se vive en los espacios sociales tiene relación con la postura que toman las figuras autoridad (como las paternas y las docentes) en relación a asumirse como figuras de autoridad, la complejidad radica en resolver el cómo erigirse tal cual en un contexto cultural en el que el imperativo categórico ${ }^{2}$ del respeto a la ley y a las figuras de autoridad se ven rebasadas en la práctica por las resistencias del sujeto en formación ${ }^{3}$ a sujetarse al orden social y a quienes representan la autoridad. Esta resistencia es cada vez más característica de la condición cultural que estamos viviendo y a la que se le denomina posmodernidad, cuyo rasgo primordial es la declinación de los grandes relatos: Los grandes íconos representativos de los valores sociales pierden cada vez mayor fuerza, entonces, el sujeto queda expuesto a las exigencias sociales y materiales de una cultura pero sin la fortaleza que le daba el estar sostenido por un entramado de preceptos morales y éticos necesarios que regulan al sujeto y a su relación con el otro, que es la ley a la que el sujeto es amarrado.

La tarea es construir la paz social cuando el sujeto se des-sujeta de la ordenanza social. No podemos conseguir esa paz interior y seguridad de forma autónoma ni en soledad, necesariamente necesitamos contar con el otro y tenerle instalado como un semejante al que debe considerar con sus mismas prerrogativas, condición que se construye en el proceso de formación del sujeto y que es regulado a través del sometimiento a la ley familiar y social, lo que solo se puede dar bajo el cobijo de la educación.

\section{EL DIÁLOGO:}

\section{MEDIO Y FIN DE LA EDUCACIÓN PARA LA PAZ Y LA DEMOCRACIA}

Educar para la paz y para la democracia tiene en la propiciación y construcción del diálogo un espacio social favorable a la posibilidad de que el sujeto dé cabida a la escucha del otroy con ello, a que en su propio mundo de la vida conceder un espacio al semejante, en esto, el lenguaje es la vía ya que los seres humanos lo usamos para representar el mundo de formas personales, propias de cada quien.

Es por ello que considero que algo importante de plantear en las sugerencias para el trabajo a favor de la educación para la paz y la democracia es el abordaje de los significantes y significados de violencia e intolerancia en el lenguaje

${ }^{2}$ El Imperativo Categórico un concepto kantiano referido a un mandato volitivo moral a priori con carácter universal y necesario, es decir, es un obrar de acuerdo a un "deber ser" o "deber hacer"; su cualidad de ser adquiridas a posteriori, ni guiada por el deseo o propósito. Mandamiento autónomo, esto es, independiente de religión o ideología alguna y capaz de regir el comportamiento humano en todas sus manifestaciones. ${ }_{3}^{3}$ Sujeto en proceso de socialización y de desarrollo psicosocial. 
y la propuesta es LA ACCIÓN COMUNICATIVA PARA LA REDEFINICIÓN DEL LENGUAJE VIOLENTO A FAVOR DEL LENGUAJE PACÍFICO.

Para ello es necesario reconstruir un espacio de entendimiento a través de la expresión de los sujetos, del lenguaje y la comunicación, en busca de la tolerancia y solidaridad, como basamento de un nuevo pacto social. Al respecto, Rosa María Armendáriz (Ortiz, 2012: 386) cita a Habermas (1981) al explicar que:

"El lenguaje, como acción comunicativa, prefigura una sociedad reflexiva, que se une por el conocimiento y no por la imposición o el temor. En el lenguaje, afirma, está la base de la democracia, porque permite una comunicación e interacción eficaz, equilibrada y libre.

La acción comunicativa, bajo el aspecto funcional de entendimiento, sirve a la tradición y a la renovación del saber cultural; bajo el aspecto de coordinación de la acción, sirve a la integración social y a la creación de solidaridad; y bajo el aspecto de socialización, sirve a la formación de identidades personales. A estos procesos de reproducción cultural, integración social y socialización, corresponden los componentes estructurales del mundo de la vida que son la cultura, la sociedad y la personalidad"

Para Habermas (1984) la acción comunicativa ayuda en el desarrollo de una democracia deliberativa y participativa.

Se requiere fomentar un diálogo efectivo, de verdadera escucha del otro, en los temas fuente o foco de tensión, de conflicto, de altercado, se trata de, llegar al punto de que los sujetos tengan la capacidad y predisposición al diálogo argumentado en la búsqueda de alternativas de solución; "la acción comunicativa presupone dos sujetos capaces de lenguaje y acción que buscan entenderse mediante actos de habla para coordinar de común acuerdo sus interpretaciones y acciones" (Ortiz, 2012: 387).

\section{CONCLUSIONES}

El mundo de hoy día plantea un duro reto a la convivencia, ya que la condición posmoderna parece estar dando un espacio privilegiado a la construcción de condiciones personales individualistas, egocéntricas y narcisistas, es por ello que esta realidad nos precisa a insistir en la búsqueda de la escucha al otro, para la construcción de diálogos de argumentación tan racionales (en donde la lógica sea capaz de revisar las premisas de la construcción de los juicios con sensatez y sensibilidad aplicada al rescate del interlocutor) como respetuosos de las diferencias e, idealmente de la búsqueda de afinidades donde el principal consenso sea el respeto a la historia y al contexto de cada cual.

Sin embargo, habitar en una sociedad más tolerante y pacífica no se ha se dar por sí solo, es una tarea que reclama el dejar de apostarle a la búsqueda incesante del encuentro de los goces en el vínculo con los objetos que debiera darse en términos de mayor compromiso para lograr metas afines al desarrollo comunitario, dándole menos espacio al tipo de prácticas sociales cargadas de connotaciones perversas en donde no importa el otro en nombre del mínimo esfuerzo para el logro de la resolución del mundo de la vida cotidiana que la sociedad neoliberal define.

Construir este tipo de vínculos de respeto al otro y de compromiso social para ganar tolerancia y paz en la dinámica global a través de la convivencia, solo puede ser posible mediante un concienzudo proceso formativo tomado por la ética para sostener la legalidad y sobre todo, la justicia.

Al respecto, habremos de tener claro que la base para una sociedad democrática y pacífica es la convivencia regulada por principios categóricos enmarcados éticamente en un entramado interdependiente de líneas de acción que corresponden a la tolerancia, la legalidad y los derechos humanos.

\section{BIBLIOGRAFÍA}

Aguilera, Beatriz; Cascon, Paco; Bastida, Anna. (2000) Colección Edupaz, dirigida por el seminario de Educación para la paz de la Asociación. Editorial Los libros de la catarata. Madrid, .

Cilano Peláez, Johanna; Córdova Jaimes, Edgar y Chaguaceda, Armando "Participación ciudadana y reforma del Estado en Venezuela: entender la política a través del ciudadano" en OSAL (Buenos Aires: CLACSO) Año X, № 26, octubre. 2009.

Cunill, Nuria. Participación ciudadana: dilemas y perspectivas para la democratización de los Estados latinoamericanos. Centro Latinoamericano de Administración para el Desarrollo. Caracas. 1991 
Habermas, Jürgen. Teoría de la acción comunicativa, I. Racionalidad de la acción y racionalización social. Tauros Humanidades. Madrid. 1994

Habermas, Jürgen. "Tres modelos de democracia. Sobre el concepto de una política deliberativa". Polis, Revista de la Universidad Bolivariana. 2005.

Ortega Ruiz, Pedro. 2004. "Educar para la participación ciudadana". Pedagogía Social. Revista Interuniversitaria, num. Diciembre-Sin mes, pp. 215236.

Monclús, Antonio y Saban, Carmen (coordinadores). Educación para la Paz, Ediciones CEAC, Barcelona, 2008.

Solís, Luis Guillermo; Peñas, Mercedes. Educación para la paz. San José: EUNED, 1995.

Tuvilla Rayo, José. Educar en los derechos humanos. Editorial CCS, Madrid, 1993.

Ortiz Marín, Celso, et.al. Diagnóstico territorial de las causas sociales de las violencias: el caso de Guasave, Sinaloa. Universidad Autónoma Indígena de México. México, 2012.

\section{Pedro Ceballos Rendón}

ceballos_rpedro@hotmail.com

Lic. en Psicología Social por la UANL, estudiante del posgrado en Estudios para la Paz, la Interculturalidad y la Democracia en la UAIM, ensayista e investigador en las ciencias sociales en temas como la prevención de riesgos psicosociales, la participación ciudadana, la cultura sociopolítica y la seguridad pública. Encargado del diseño del Diplomado para maestros de educación básica: Acompañamiento para la construcción de ambientes escolares libres de violencias. 